\title{
Cost-Effectiveness of Baricitinib for Patients with Moderate-to-Severe Rheumatoid Arthritis After Methotrexate Failed in China
}

\author{
SiNi Li $\cdot$ JianHe Li $\cdot$ LiuBao Peng $\cdot$ YaMin Li $\cdot$ XiaoMin Wan
}

Received: March 9, 2021 / Accepted: April 9, 2021 / Published online: April 24, 2021

(C) The Author(s) 2021

\begin{abstract}
Introduction: A phase 3 (RA-BEAM study) clinical trial reported that baricitinib $(\mathrm{BCT})+$ methotrexate (MTX) had clinical improvement compared with adalimumab (ADA) + MTX as a first-line strategy in patients with rheumatoid arthritis (RA) who had inadequate responses to MTX monotherapy. However, from the perspective of the Chinese healthcare system, the cost-effectiveness of introducing BCT into current treatment for patients with RA unresponsive to MTX remains unclear.
\end{abstract}

Methods: A patient-level microsimulation model was used to extrapolate the lifetime

Supplementary Information The online version contains supplementary material available at https:// doi.org/10.1007/s40744-021-00308-w.

SiN. Li · Y. Li ( $₫)$

Clinical Nursing Teaching and Research Section, The Second Xiangya Hospital, Central South University, Changsha 410011, China

e-mail: aminny@csu.edu.cn

SiN. Li · Y. Li

The Xiangya Nursing School, Central South

University, Changsha 410013, China

J. Li · L. Peng · X. Wan $(\varangle)$

Department of Pharmacy, The Second Xiangya

Hospital, Central South University, Changsha

410011, China

e-mail: wanxiaomin@csu.edu.cn incremental cost per quality-adjusted life-year (QALY) and other outcomes. This study compared treatment sequences with or without firstline BCT with current treatment sequences, including adalimumab, etanercept, tocilizumab, and palliative care. Effectiveness and physical function were assessed using the American College of Rheumatology (ACR) 20/50/70 response and Health Assessment Questionnaire (HAQ). The input parameters of the model, comprising patient characteristics (sex and age) and treatment efficacy (ACR responses and HAQ score), were derived from a phase III clinical trial and network meta-analysis. The total cost estimation included direct costs and indirect costs. Probabilistic and univariate sensitivity analyses were performed, as were a series of scenario analyses.

Results: The lifetime analysis revealed that adding BCT as a first-line treatment resulted in a QALY gain of 2.66 years; this gain would cost an incremental $\$ 26,662$, leading to an incremental cost-effectiveness ratio of $\$ 10,036 /$ QALY per patient compared with the current treatment sequence. Sensitivity and scenario analyses showed the results to be robust.

Conclusions: From a Chinese payer perspective, the introduction of BCT into the current treatment sequence is projected to be a cost-effective option as first-, second-, third-, and fourth-line treatment for patients with moderate-to-severe RA. 
Keywords: Rheumatoid arthritis; Baricitinib; Cost-effectiveness analysis; Model; Janus kinase inhibitors

\section{Key Summary Points}

\section{Why carry out this study?}

Approximately 4 million people live with rheumatoid arthritis (RA) in China, with an illness cost of $\$ 3826 /$ patient annually, resulting in a heavy financial burden for RA patients

The introduction of novel drugs into the Chinese market brings another option for RA patients, but its cost-effectiveness remains unknown

From the perspective of the Chinese healthcare system, whether adding baricitinib to the current treatment sequence is cost-effective for patients with moderate-to-severe rheumatoid arthritis in whom methotrexate therapy failed is investigated.

\section{What was learned from the study?}

Adding baricitinib as a first-line treatment resulted in an incremental costeffectiveness ratio of $\$ 10,036 /$ qualityadjusted life-years per patient and had a $100 \%$ likelihood of being cost-effective if the willingness-to-pay threshold was $>$ $\$ 16,430$ compared with the current treatment sequence

From a Chinese payer perspective, the introduction of baricitinib into the current treatment sequence is projected to be a cost-effective option for patients with moderate-to-severe rheumatoid arthritis.

\section{DIGITAL FEATURES}

This article is published with digital features, including a summary slide to facilitate understanding of the article. To view digital features for this article go to https://doi.org/10. 6084/m9.figshare.14381429.

\section{INTRODUCTION}

Rheumatoid arthritis (RA), a chronic, debilitating and systemic autoimmune disease, brings substantial economic burdens to patients with RA and affects their quality of life. In China, the prevalence of RA is $0.28 \%$ [1], indicating that there are $>4$ million RA patients; the male:female ratio is 1:4 [2]. As the clinical remission rate of RA patients is only $8.6 \%$, there is a high rate of disability, at $50.3 \%$ [3]. Overall, RA has become the leading cause of disability and a major public health problem in China [4].

For patients with active RA, the most commonly prescribed agent is the conventional disease-modifying antirheumatic drug (cDMARD) methotrexate (MTX) [5]. However, because of poor tolerability and inadequate efficacy, MTX use is limited [6]. The American College of Rheumatology (ACR) and European League Against Rheumatism both recommend that biologic treatment be initiated in patients with RA who have a suboptimal response to cDMARDs [5, 7]. Consequently, biologic DMARDs (bDMADs), including the tumor necrosis factor inhibitors (TNFis) etanercept, infliximab, adalimumab, golimumab, and certolizumab, are widely employed in clinical practice [8]. Although the use of biologic agents has contributed significantly to effective disease control, studies have found that many patients have poor adherence and active disease and discontinue treatment because of severe adverse events (SAEs), intolerance, or other factors $[9,10]$.

The Janus kinase (JAK) signaling pathway is implicated in the pathogenesis of RA [11]. JAK inhibitors are collectively referred to as targeted DMARDs (tDMARDs) with bDMARDs [12]. Recent studies have demonstrated that compared to biologic inhibitors, JAK inhibitors result in greater clinical improvement or are at least non-inferior $[13,14]$. BCT, an oral reversible and selective tyrosine-protein inhibitor of JAK1/JAK2 [15], was approved by the US Food 
and Drug Administration (FDA) in July 2018. Three clinical trials have shown the efficacy of BCT with or without MTX in patients naïve to cDMARDs and in those with intolerance to or an inadequate response to both cDMARDs and bDMARDs [16-18]. Recently, the RA-BEAM study, a phase 3 , randomized, double-blind, placebo- and active-controlled clinical trial, compared baricitinib (BCT) + MTX with adalimumab (ADA) + MTX as a first-line strategy in patients with RA who had inadequate responses to MTX monotherapy [14]. The results of the study confirm the findings of other trials of BCT therapy [18], both supporting that BCT is associated with significant clinical improvement and introducing a new agent to the clinical treatment sequence, which may broaden treatment options for RA patients and rheumatologists [14].

Although the previous study of Max Schlueter et al. indicated that BCT is a cost-effective strategy compared with ADA for Spanish patients with active RA and that of Celine reached the same conclusion for patients with refractory RA in The Netherlands, the cost-effectiveness of BCT + MTX relative to ADA + MTX has not been assessed in China $[19,20]$. New agents often come with a financial burden, even though they might improve the clinical outcome for patients [21, 22]. Therefore, it is necessary to assess the economic value of BCT for patients with moderate-to-severe RA, as BCT was approved by the Chinese National Medical Products Administration (CNMPA) in June 2019. Therefore, the aim of this study was to estimate from the perspective of the Chinese healthcare system the cost-effectiveness of adding BCT to the current treatment sequence for patients with moderate-to-severe RA in whom MTX therapy failed.

\section{METHODS}

\section{Model Structure Overview}

To best reflect clinical practice, a microsimulation model in which patients transitioned through a predefined treatment sequence was constructed on the basis of individual patient- level simulation using Treeage Pro software (https://www.treeage.com/). The model tracked patients with different population characteristics over a lifetime horizon (50 years), with the follow-up time separated into 6-month cycles. Patients were moved through the treatment sequence and finally progressed into the palliative care (PC) phase according to the ACR criterion and discontinuation probability. Mortality risk, utility values, and hospitalization costs in every cycle were calculated based on HAQ scores. Health-related costs and quality of life were evaluated throughout the patient's lifetime. Incremental cost, QALYs, and the incremental cost-effectiveness ratio (ICER) are presented as the main outcomes of the cost-effectiveness analysis.

\section{Patient Baseline Characteristics}

A total of 50,000 patients with moderate-tosevere RA who had an inadequate response to MTX were simulated in the analysis. All eligible patients were enrolled on the basis of the RABEAM study population, mainly including parameters of age, sex, and baseline HAQ [14]. The mean patient weight of the Chinese general population was used because of the lack of data in the RA-BEAM trial [23]. Details of the patients' characteristics are presented in Table 1. This article is based on previously conducted studies and does not contain any new studies with human participants or animals performed by any of the authors.

\section{Treatment Sequence of RA Patients}

The current treatment sequence (comparator group) of the study and comparator groups we set for RA patients in China was obtained based on the 2018 RA treatment guideline in China and the expert opinion of four rheumatologists from three tertiary hospitals [24]. In the comparator group, TNFi bDMARDs (i.e., ADA) were used as first-line treatment when patients were unresponsive to cDMARDs [22]. After failure of the first-line treatment, patients were switched to another TNFi (i.e., etanercept) as second-line treatment [25]. After etanercept failure, it was 
Table 1 Key model parameters

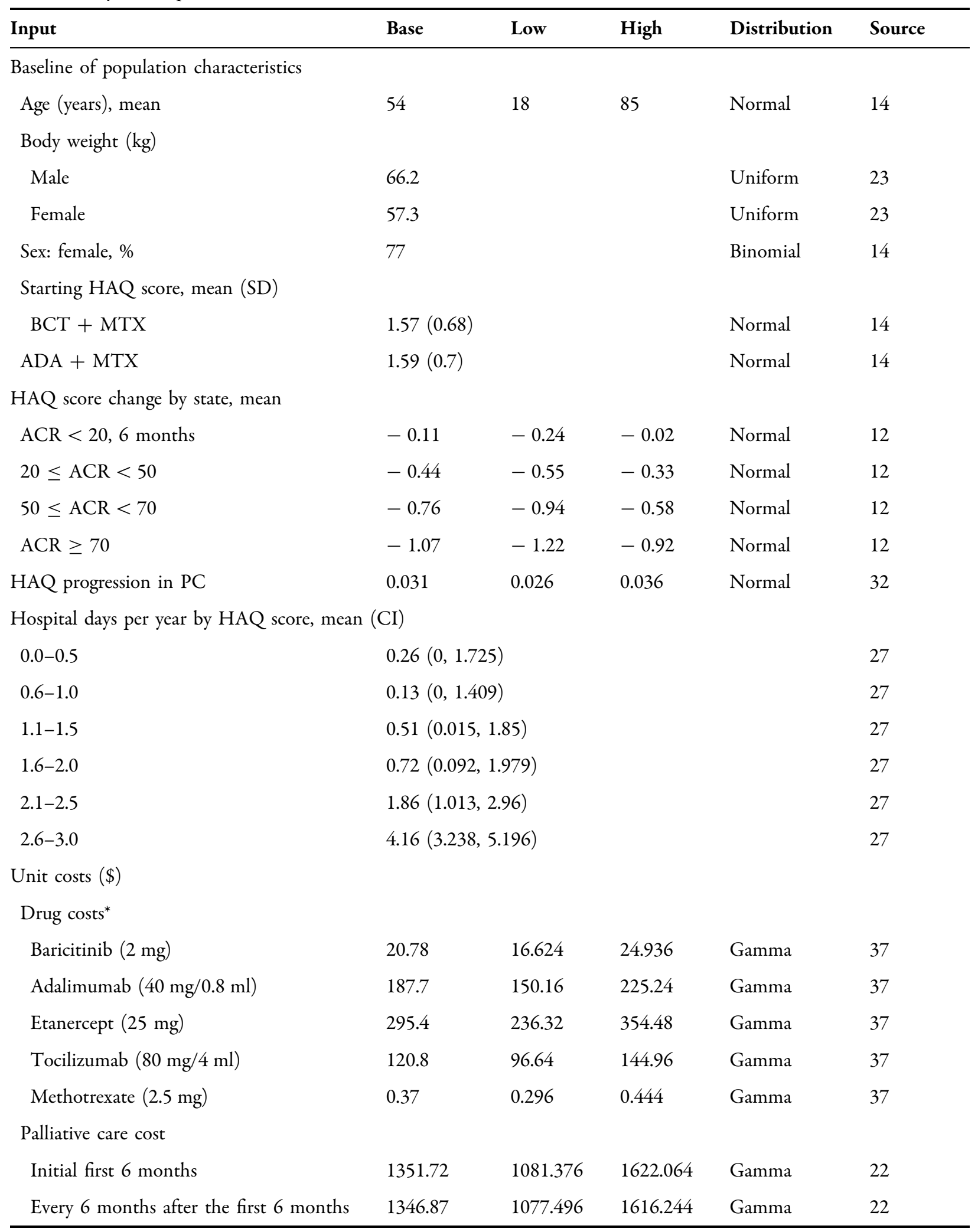


Table 1 continued

\begin{tabular}{llllll}
\hline Input & Base & Low & High & Distribution & Source \\
\hline Administration cost & & & & & \\
IV & 8.95 & 7.16 & 10.74 & Gamma & Expert panel \\
SC & 0.58 & 0.464 & 0.696 & Gamma & Expert panel \\
Inspection cost & & & & & \\
$\quad$ Initial first 6 months & 162.82 & 130.256 & 195.384 & Gamma & 22 \\
$\quad$ Every 6 months after the first 6 months & 74.09 & 59.272 & 88.908 & Gamma & 22 \\
Hospitalization, per day & 166 & 83.26 & 208.15 & Gamma & Expert panel \\
Outpatient follow-up visit, per cycle & 88.79 & 40.76 & 111.06 & Gamma & Expert panel \\
Average salary, per day & 19.76 & 5.51 & 24.17 & Gamma & 39 \\
SAEs & & & & & \\
Probability of serious infection (\%) & & & & & 12 \\
cDMARDs or PC & 0.0382 & 0.025 & 0.0533 & Normal & 22 \\
tDMARDs & 0.0856 & 0.062 & 0.1089 & Normal & 12 \\
Treatment of pneumonia (\$) & 1761.4 & 1409.12 & 2113.68 & Gamma & \\
Mortality risk for RA, per HAQ unit & 1.33 & 1.10 & 1.61 & & Uniform \\
Disutility of SAEs & 0.156 & - & - & & 42 \\
\hline
\end{tabular}

$S D$ standard deviation, $C I$ confidence interval, $B C T$ baricitinib, $A D A$ adalimumab, $M T X$ methotrexate, $A C R 20$ American College of Rheumatology 20\% improvement criteria, ACR50 ACR 50\% improvement criteria, ACR70 ACR 70\% improvement criteria, $H A Q$ Health Assessment Questionnaire, $P C$ palliative care, $I V$ intravenous injection, $S C$ subcutaneous injection, $c D M A R D s$ conventional disease-modifying antirheumatic drugs, tDMARDs targeted disease-modifying antirheumatic drugs, $R A$ rheumatoid arthritis, $S A E s$ severe adverse event

*Baricitinib: $4 \mathrm{mg}$ orally once daily; adalimumab: $40 \mathrm{mg}$ subcutaneously every other week; etanercept: $50 \mathrm{mg}$ subcutaneously once a week; tocilizumab: $8 \mathrm{mg} / \mathrm{kg}$ intravenously once every 4 weeks; methotrexate: $10 \mathrm{mg}$ orally twice a week

assumed that patients would be treated with non-TNFi biologics (i.e., tocilizumab), which is another class of biologic agents with different mechanisms of action [24, 26]. After failure of third-line treatment, patients were eventually switched to the PC phase, mainly comprising MTX, cyclosporine, hydroxychloroquine, leflunomide, azathioprine, sulfasalazine, celecoxib, and prednisolone, until death [22] (Fig. 1).

To understand the comprehensive cost-effectiveness of adding BCT to the current treatment sequence for patients with moderate-tosevere RA in China, a base case and three scenario analyses were performed. In the base case analysis, the study group was administered BCT before the current treatment sequence as a firstline treatment, and the current treatment sequence was used as a comparator group. Three scenarios were then separately compared with the comparator group: (1) BCT after ADA as a second-line therapy; (2) BCT after second-line treatment with TNFi (i.e., etanercept) as thirdline therapy; (3) BCT after non-TNFi biologic (i.e., tocilizumab) as fourth-line therapy. The model assumed that all treatments were administered in combination with MTX. 


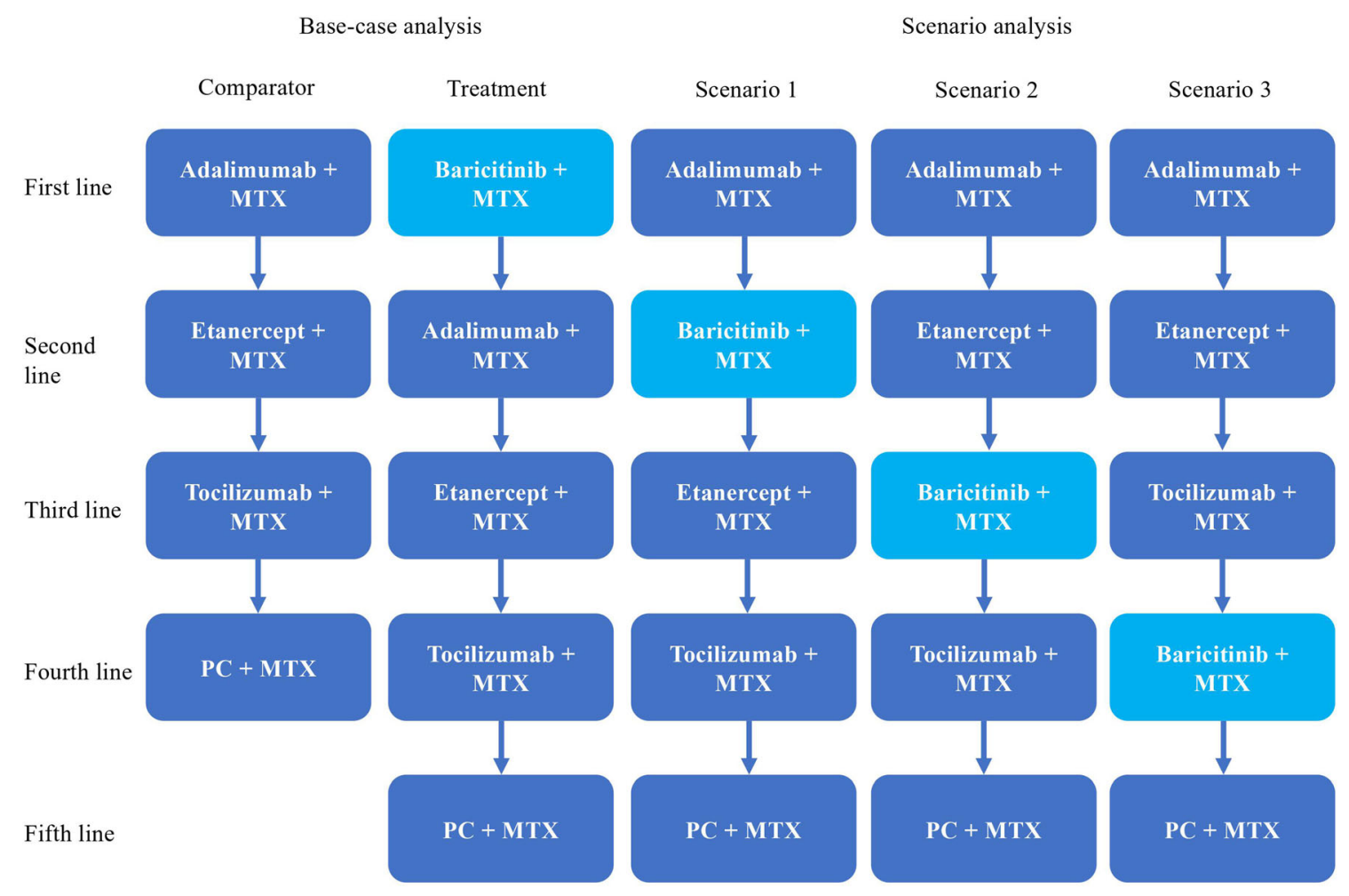

Fig. 1 Treatment sequence. $P C$ palliative care, $M T X$ methotrexate

\section{Treatment Response and Discontinuation}

The core of the model is treatment response measured using the ACR criteria, classified as non-responders (not achieving ACR 20\% improvement criteria), ACR 20 (achieving ACR $20 \%$ improvement criteria), ACR 50 (ACR 50\% improvement criteria), or ACR 70 (ACR 70\% improvement criteria). A decision to remain on current treatment (maintenance phase) or switch to the next treatment is determined by identifying whether the patients achieve ACR 20 or above ACR20 (i.e., ACR50 or ACR70) at the end of the initial cycle of every treatment line (i.e., BCT, ADA, etanercept, and tocilizu$\mathrm{mab}$ ); otherwise, the patient will move to the next treatment line $[22,27]$. Therefore, ACR20 was applied to estimate the discontinuation probability of the initial cycle of each treatment line. The process was repeated until the RA patient was switched to the PC phase. Treatment responses for BCT and ADA were derived from the RA-BEAM study, and etanercept and tocilizumab were obtained from a Bayesian network meta-analysis that included 96 unique random controlled trials (RCTs) of RA treatment to estimate the treatment effects of anti-rheumatic drugs in the initial cycle $[12,14,20]$ (Supplementary Material Table 1).

After the initial cycle of every treatment line, responders who at least attained ACR20 continued on the current treatment but experienced a discontinuation of treatment due to lack of efficacy or intolerance. Specifically, the discontinuation probability of other cycles was based on a survival curve obtained from the CORRONA database using a lognormal model $[28,29]$. A treatment discontinuation curve was generated, and the subsequent discontinuation probability was calculated from a lognormal distribution to estimate a transition from one treatment to another for long-term progression (Supplementary Material Figure 2 and Supplementary Material Table 2).

In addition to lack of response, severe adverse events (SAEs) may also lead to treatment switching $[22,26,27]$. Based on a previous study, we only included severe infection (i.e., 


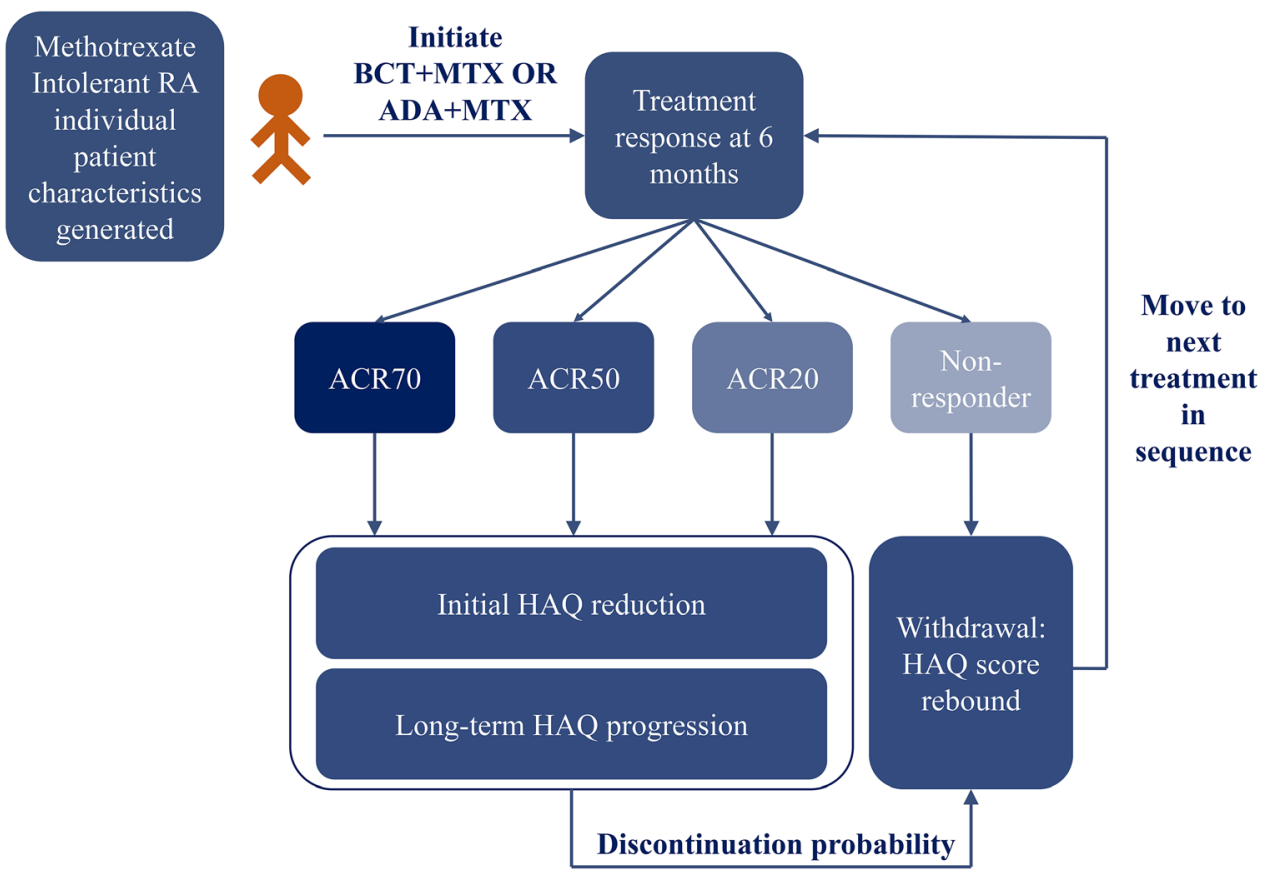

Fig. 2 Overview of the RA lifetime model. The schematic illustrates how treatment impacts the HAQ score within the model. $B C T$ baricitinib, $A D A$ adalimumab, $M T X$

pneumonia) in the model as an SAE [12]. The probabilities of serious infection are presented in Table 1.

\section{Change in HAQ}

The Health Assessment Questionnaire (HAQ) is an instrument for estimating the physical function of patients, with impacts on the patient mortality rate, health utility and hospitalization rate in the model. The HAQ score ranged from 0 to 3 in multiples of 0.125 , whereby a higher score indicated a worse state of disease [30]. Therefore, change in the HAQ score was the prime driver of both cost and benefit in the model [25]. The microsimulation model allows HAQ changes over time to simulate disease progression for each patient. The four response levels were associated with different changes in the HAQ score. The relationship between the change in HAQ score and ACR response rate at the initial 6 months for tDMARDs was derived from a previous cost-effective study of RA [27] (Table 1 and Fig. 2). If a methotrexate, ACR American College of Rheumatology, $H A Q$ Health Assessment Questionnaire

patient responds to treatment, then the HAQ score of the treatment will be decreased, indicating disease improvement [31]. The change in HAQ score in the initial cycle of every treatment line was subtracted from the baseline HAQ score, and the HAQ change of other cycles was subtracted from the HAQ score of the last cycle to simulate the influence of therapy on disease progression. Similar to other studies, we assumed that the change in HAQ score of the response level at the initial 6 months was the same for all tDMARDs [12, 27]. After the initial cycle of every treatment line, except for the PC phase, the change in HAQ score is assumed to be constant (no disease progression in HAQ) for the long-term period if the patient remains on the current treatment, which was tested in a sensitivity analysis $[12,27]$. In the PC phase, we assumed that the HAQ score progressed at a constant linear rate (increase of 0.031 every 6 months) over time [32].

Any improvement in the HAQ score for patients who quit the current treatment and move to next treatment will be lost, and they will experience a rebound in the HAQ score 
before starting the next treatment within the predefined sequence, at which point the treatment process commences again [25]. We assumed that the HAQ score would increase back to the beginning of the first cycle for each treatment line [22].

\section{Mortality}

Death was considered to be related to the HAQ score and could occur at any time in the model [33]. The mortality rate in the model was estimated based on age-/sex-specific mortality from the Chinese life table, which was sourced from the mortality database of the World Health Organization (WHO) [34]. The equation of mortality adjustment (Eq. 1), which has been published previously, is dependent on the HAQ score [22]:

$$
\begin{aligned}
& \text { RA-specific adjusted mortality } \\
& \quad=\text { mortalityrate from life table } \times 1.33^{\mathrm{HAQ}}
\end{aligned}
$$

\section{Utility}

Health-related quality of life of the RA patients for each cycle was calculated by using a previously published equation that is widely utilized by economic evaluation analysis in RA and shows great value for moderate-to-severe RA $[27,35]$. Equation 2 below mapped the HAQ score into the EuroQol five-dimensional questionnaire (EQ-5D).

$$
\begin{aligned}
\mathrm{EQ}-5 \mathrm{D}= & 0.82 \\
& -(0.11 \times \mathrm{HAQ})-\left(0.07 \times \mathrm{HAQ}^{2}\right)
\end{aligned}
$$

Grade $3 / 4$ AEs with a frequency $\geq 5 \%$ or a difference of $>2 \%$ between treatments in the RA-BEAM trial were included in our study. Therefore, we only considered pneumonia as an SAE in the model because the safety profiles of tDMADRDs are similar and would not significantly alter the results [12]. Based on a prior study, we assumed that disutility caused by severe infection, such as pneumonia, was decreased by 0.156 units of utility for the duration of the month of infection [36].

\section{Resource Use and Costs}

In this study, we included in the model direct medical costs (i.e., drug acquisition, administration, monitoring, SAE management and hospitalization) and indirect costs (i.e., productive loss) relevant to the Chinese healthcare system. Unit costs were estimated from national databases, published studies, or a Chinese expert panel. Drug acquisition costs were derived from the website of China Medical Bidding [37]. All drug agents in the model except BCT and MTX were administered subcutaneously. Monitoring costs, including chest $\mathrm{x}$-rays and physician visits, were considered to be the same in both sequences. The cost of any SAE is assumed to be equal to that of different tDMARDs and equal to the cost of pneumonia (\$1761.4). The cost of hospitalization was considered related to the HAQ score: a study from Carlson et al. [27] revealed the relationship between HAQ score and days in hospital annually. Cost per day in the hospital was derived from an expert panel. Costs were adjusted to 2019 US dollars ( 1 USD $=6.87$ RMB) [38]. According to Chinese guidelines for economic evaluation, costs and utilities were discounted at $3 \%$ per year.

\section{Validation}

All sources, inputs, and assumptions were validated by an expert panel, including four Chinese rheumatologists, one pharmacist, and two health economists. An independent modeler verified the implementation of the model. Halfcycle correction was applied for all costs and utilities by taking the average of the input parameters at the beginning and end of the cycle, considering transitions (events) that occurred halfway through a cycle.

\section{Sensitivity Analysis}

One-way sensitivity analysis was performed to determine key drivers in the model. Twenty-one variables, including drug costs, discount rate, baseline $\mathrm{HAQ}$, initial $\mathrm{HAQ}$ change in different response levels, annual HAQ score change in PC 
phase, and other costs, were varied individually to estimate the proportional effect on model outcomes. In addition, to test the robustness of the model and degree of uncertainty in our results estimates, we performed multiple scenarios that considered sources of data around key input parameters and alternative assumptions. For example, BCT was inserted at different positions in the treatment sequence to evaluate its impact on the cost-effective analysis. We also considered a series of scenarios regarding the heterogeneity of RA patients (Supplementary Material Table 4; Part B).

Probability analyses were conducted for 2000 sets of 2000 patients to assess overall parameter uncertainty. Key input parameters were simulated from parametric distribution to generate 2000 estimates of the costs and benefits in each strategy. All costs followed gamma distributions; age, baseline HAQ, and HAQ score changes followed normal distributions. Discontinuation probabilities followed beta distributions, weight and disutility of SAEs followed uniform distributions, and sex followed binomial distribution. In this study, willingness to pay (WTP) was set at $\$ 30,950 /$ QALY in China by using three times the per capita gross domestic product, as recommended by WHO guidelines [39]. If the result of ICER we estimated was lower than the WTP in China, then it could be considered that the study group was cost-effective compared with the current treatment sequence.

\section{RESULTS}

\section{Base Case Analysis}

A 50,000 Monte Carlo simulation was used to conduct base case analysis, and the results are displayed in Table 2. The base case analysis indicated that compared with the comparator group, the study group provided more accumulated QALYs, 12.26 versus 9.61 QALY (difference, 2.66 QALY), but was also more costly (incremental cost: \$26,662) over the lifetime period. The ICER of the study group vs. comparator group (i.e., 10,036) was below the one time per capita GDP (i.e., \$10,316/QALY),
Table 2 Base case analysis results: total costs, QALYs, and ICERs

\begin{tabular}{llll}
\hline & BCT-MTX & ADA-MTX & Incremental \\
\hline Total cost, \$ & 95,820 & 69,158 & 26,662 \\
QALYs & 12.26 & 9.61 & 2.66 \\
ICER, \$ & - & - & 10,036 \\
\hline
\end{tabular}

$B C T$ baricitinib, $A D A$ adalimumab, $M T X$ methotrexate, $I C E R$ incremental cost-effectiveness ratio, $Q A L Y$ qualityadjusted life-year

which was recommended by the WHO as a "very cost-effective" threshold [40].

\section{Sensitivity and Scenario Analyses}

The results of the model were most sensitive to age, drug cost of BCT and etanercept, discount rate, and probability of serious infection in the PC phase. The cost-effectiveness acceptability curve showed that the study group was cost-effective under the Chinese threshold. Moreover, the study group had a 90\% and 100\% likelihood of being cost-effective if the WTP was $>\$ 13,660$ and $\$ 16,430$ compared with the current treatment sequence (Fig. 3 ).

Scenario analyses of sequences, including BCT added to the comparator group as second-, third-, and fourth-line treatment, also revealed improved costs and QALY gains compared with the comparator group: ICERs of $\$ 10,619$; $\$ 11,789$, and $\$ 10,820$ per QALY estimated for the administration of BCT as a second-, third-, and fourth-line treatment, respectively. According to all scenario analyses of sequences, adding BCT to the comparator group as a second-, third-, and fourth-line treatment was < $\$ 30,950 /$ QALY and likely to be cost-effective in China compared with the comparator group (Supplementary Material Table 3; Part A). Furthermore, we used statistical methods to explore whether there was statistical difference between each scenario. The results demonstrated that although administrating BCT as second-line therapy had the lowest ICER compared with those in all scenario analyses of 


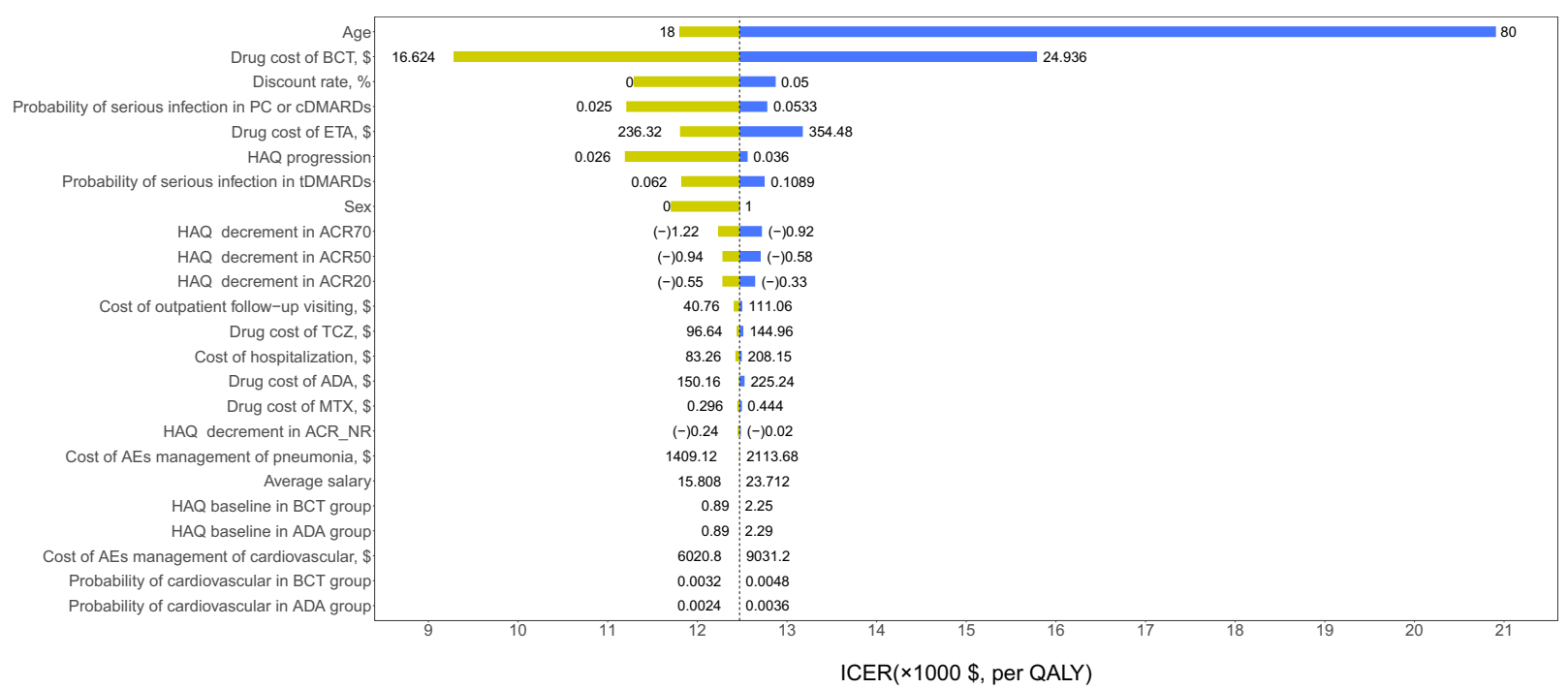

Fig. 3 Tornado diagram for one-way sensitivity analysis. $B C T$ baricitinib, $A D A$ adalimumab, MTX methotrexate, $E T A$ etanercept, INF infliximab, ACR20 American College of Rheumatology $20 \%$ improvement criteria, $A C R 50$ ACR 50\% improvement criteria, $A C R 70$ ACR 70\% improvement criteria, $N R$ no response, $H A Q$ Health

sequences, there was no statistical difference related to second-line therapy compared to other scenarios (Supplementary Material Table 4; Part A).

Part B illustrated that the cost-effectiveness of the study group was $<\$ 30,950 /$ QALY for all scenarios, though we set the baseline HAQ of the study group at the upper limit $(\mathrm{HAQ}=2)$. For example, even if the age of patients was set at the upper limit (age $=80$ years), the ICER for the BCT + MTX strategy was still $<\$ 30,950 /$ QALY.

\section{DISCUSSION}

To our knowledge, this is the first study to evaluate the cost-effectiveness in China of introducing BCT to adalimumab-etanercepttocilizumab-PC (current treatment sequence) for patients with moderate-to-severe RA in whom MTX is considered inappropriate. To fill this evidence gap, we developed a cost-effectiveness model by adopting a microsimulation approach that models individual patients to account for heterogeneity instead of a patient
Assessment Questionnaire, $P C$ palliative care, $H A Q$ progression annual HAQ score change in $\mathrm{PC}$ phase, AEs adverse events, $c D M A R D s$ conventional disease-modifying antirheumatic drug, $t D M A R D s$ targeted disease-modifying antirheumatic drug

cohort. The topic of this study has relevance for patients, clinicians, and policy makers. RCTs have suggested that BCT results in a significant clinical improvement compared with ADA for patients with moderate-to-severe RA, despite previously receiving treatment with MTX. Our study compared a series of treatment sequences that introduced BCT into current clinical practice for RA, providing a comprehensive RA treatment guideline for clinical use. Specifically, our study found that adding BCT to the current treatment sequence was cost-effective compared with the comparator group alone.

Due to the different model settings and input parameters (i.e., drug acquisition cost, treatment sequence, and time horizon), the results of our study were inconsistent with those of evaluation analyses of BCT compared with biologics in other countries. For example, a study in Spain conducted by Schlueter et al. [20] revealed that a BCT first-line strategy was associated with 0.09 QALYs gained over a lifetime horizon, with an incremental cost of $-€ 558$ versus ADA first-line treatment. BCT is a costeffective option for patients with moderate-to- 
severe RA in whom there is an inadequate response to cDMARDs. Another study from The Netherlands developed by Celine estimated the ICER to be $-€ 238,418$, which suggested that the use of BCT instead of ADA in the treat-totarget approach is cost-effective for refractory RA patients [19]. The negative ICER in the cases above confirms the superiority of the BCT strategy: it accumulates higher QALYs with lower cost over the model time horizon.

This study had several limitations, primarily because of the inherent shortcomings of the RCT in terms of the follow-up period. Most data we obtained from the RCT and NMA in the model were for approximately 12-24 months, but the time horizon of the model was the lifetime, and short-term clinical data may introduce some biases in extrapolating longterm efficacy and costs. Indeed, inadequate information about HAQ progression for the long-term period ( $>24$ months on treatment) led to an assumption that the HAQ changed constantly. This is an unavoidable limitation for most RCTs because conducting long-term follow-up is resource intensive and prohibitively expensive. Thus, we built a decision analytic model and made reasonable assumptions to improve the robustness and practicality of the model. Second, the population characteristics of RA patients were taken from the RA-BEAM trial because of the lack of a head-to-head study that directly compared BCT + MTX with ADA + MTX in China. However, sensitivity analysis revealed that population characteristics only marginally impact the model results. Third, patients in the treatment sequence in this study were switched to PC for the remainder of their lifetime (model time horizon); this is justifiable and widely used by published articles on RA economics evaluation [12, 22]. Nonetheless, the clinical treatment sequence will have more permutations and combinations than the limited treatment sequence in this study. Fourth, according to previous studies, we assumed that only serious infection had a significant impact on the results in the model $[41,42]$. However, in real life, patients may experience different AEs; regardless, because the safety profiles of bDMARDs are similar [27], adding other AEs to the model would not alter the results. The results of sensitivity analyses also confirmed that the impact on bDMARDs caused by AEs was insignificant. Finally, we did not include treatment with BCT monotherapy, which has significant clinical improvement and may be beneficial for patients with RA who are intolerant to cDMARDs. The economic evaluation of BCT monotherapy compared to BCT + cDMARDs needs to be assessed in future studies.

\section{CONCLUSIONS}

From the Chinese payer perspective, the introduction of BCT to the current treatment sequence for treating patients with moderateto-severe RA for whom MTX fails is a cost-effective treatment option. Moreover, cost-effectiveness was obtained regardless of the position of BCT within the treatment sequence. Compared with its addition at subsequent positions in the treatment sequence, addition of BCT as a first-line treatment is the best option.

\section{ACKNOWLEDGEMENTS}

Funding. This study and the associated journal's Rapid Service Fee were funded by the National Natural Science Foundation of China (No: 71874209) and Hunan Provincial Natural Science Foundation of China (no. 2019JJ40411).

Authorship. All named authors meet the International Committee of Medical Journal Editors (ICMJE) criteria for authorship for this article, take responsibility for the integrity of the work as a whole, and have given their approval for this version to be published.

Author Contributions. Sini Li constructed the model, collected and analyzed data, and drafted the manuscript. Xiaomin Wan conceptualized the study and provided the model framework. Yamin Li contributed to the revision of the manuscript. Liubao Peng and Jianhe $\mathrm{Li}$ was the guarantor of the study and given 
technical and material support. All authors gave final approval for the version to be published.

Disclosures. Sini Li, Jianhe Li, Liubao Peng, Yamin $\mathrm{Li}$, and Xiaomin Wan have nothing to disclose.

Compliance with Ethics Guidelines. This is a model-based economic evaluation which the patient data were all obtained from previous published studies and does not contain any studies with animals or human particulars. Therefore, the study did not require the approval of the independent ethics committee.

Data Available. The datasets generated during and/or analyzed during the current study are available from the corresponding author on reasonable request.

Open Access. This article is licensed under a Creative Commons Attribution-NonCommercial 4.0 International License, which permits any non-commercial use, sharing, adaptation, distribution and reproduction in any medium or format, as long as you give appropriate credit to the original author(s) and the source, provide a link to the Creative Commons licence, and indicate if changes were made. The images or other third party material in this article are included in the article's Creative Commons licence, unless indicated otherwise in a credit line to the material. If material is not included in the article's Creative Commons licence and your intended use is not permitted by statutory regulation or exceeds the permitted use, you will need to obtain permission directly from the copyright holder. To view a copy of this licence, visit http://creativecommons.org/licenses/by$\mathrm{nc} / 4.0 /$.

\section{REFERENCES}

1. Cross M, Smith E, Hoy D, Carmona L, Wolfe F, Vos $\mathrm{T}$, et al. The global burden of rheumatoid arthritis: estimates from the global burden of disease 2010 study. Ann Rheum Dis. 2014;73(7):1316-22. https://doi.org/10.1136/annrheumdis-2013204627.
2. Jin S, Li M, Fang Y, Li Q, Liu J, Duan X, et al. Chinese Registry of rheumatoid arthritis (CREDIT): II. prevalence and risk factors of major comorbidities in Chinese patients with rheumatoid arthritis. Arthritis Res Ther. 2017;19(1):251. https://doi.org/ 10.1186/s13075-017-1457-z.

3. Li ZG. A new look at rheumatology in China-opportunities and challenges. Nat Rev Rheumatol. 2015;11(5):313-7. https://doi.org/10.1038/ nrrheum.2014.218.

4. Zhang X, Mu R, Wang X, Xu C, Duan T, An Y, et al. The impact of rheumatoid arthritis on work capacity in Chinese patients: a cross-sectional study. Rheumatology. 2015;54(8):1478-87. https://doi. org/10.1093/rheumatology/kev014.

5. Singh JA, Saag KG, Bridges SL Jr, Akl EA, Bannuru RR, Sullivan MC, et al. 2015 American College of Rheumatology guideline for the treatment of rheumatoid arthritis. Arthritis Care Res. 2016;68(1): 1-25. https://doi.org/10.1002/acr.22783.

6. Agency EM. Guideline on clinical investigation of medicinal products other than NSAIDS for treatment of rheumatoid arthritis (draft). 2015. http:// www.ema.europa.eu/docs/en_GB/document_ library/Scientific_guideline/2015/06/ WC500187583.pdf. Accessed 24 Aug 2020.

7. Smolen JS, Landewe R, Bijlsma J, Burmester G, Chatzidionysiou K, Dougados $\mathrm{M}$, et al. EULAR recommendations for the management of rheumatoid arthritis with synthetic and biological diseasemodifying antirheumatic drugs: 2016 update. Ann Rheum Dis. 2017;76(6):960-77. https://doi.org/10. 1136/annrheumdis-2016-210715.

8. Wailoo AJ, Bansback N, Brennan A, Michaud K, Nixon RM, Wolfe F. Biologic drugs for rheumatoid arthritis in the Medicare program: a cost-effectiveness analysis. Arthritis Rheum. 2008;58(4):939-46. https://doi.org/10.1002/art.23374.

9. Smolen JS, Aletaha D, McInnes IB. Rheumatoid arthritis. Lancet. 2016;388(10055):2023-38. https:// doi.org/10.1016/S0140-6736(16)30173-8.

10. Rubbert-Roth A, Finckh A. Treatment options in patients with rheumatoid arthritis failing initial TNF inhibitor therapy: a critical review. Arthritis Res Ther. 2009;11(Suppl 1):S1. https://doi.org/10. $1186 / \operatorname{ar} 2666$.

11. Banerjee S, Biehl A, Gadina M, Hasni S, Schwartz DM. JAK-STAT signaling as a target for inflammatory and autoimmune diseases: current and future prospects. Drugs. 2017;77(5):521-46. https://doi. org/10.1007/s40265-017-0701-9. 
12. Incerti D, Curtis JR, Shafrin J, Lakdawalla DN, Jansen JP. A flexible open-source decision model for value assessment of biologic treatment for rheumatoid arthritis. Pharmacoeconomics. 2019;37(6):829-43. https://doi.org/10.1007/ s40273-018-00765-2.

13. Fleischmann R, Mysler E, Hall S, Kivitz AJ, Moots RJ, Luo Z, et al. Efficacy and safety of tofacitinib monotherapy, tofacitinib with methotrexate, and adalimumab with methotrexate in patients with rheumatoid arthritis (ORAL Strategy): a phase 3b/4, double-blind, head-to-head, randomised controlled trial. Lancet. 2017;390(10093):457-68. https://doi. org/10.1016/S0140-6736(17)31618-5.

14. Taylor PC, Keystone EC, van der Heijde D, Weinblatt ME, Del Carmen ML, Reyes Gonzaga J, et al. Baricitinib versus placebo or adalimumab in rheumatoid arthritis. N Engl J Med. 2017;376(7): 652-62. https://doi.org/10.1056/NEJMoa1608345.

15. Gras J. Baricitinib: JAK inhibition for rheumatoid arthritis. Drugs Today. 2016;52(10):543-50. https:// doi.org/10.1358/dot.2016.52.10.2525742.

16. Genovese MC, Kremer J, Zamani O, Ludivico C, Krogulec M, Xie L, et al. Baricitinib in patients with refractory rheumatoid arthritis. $\mathrm{N}$ Engl J Med. 2016;374(13):1243-52. https://doi.org/10.1056/ NEJMoa1507247.

17. Fleischmann R, Schiff M, van der Heijde D, RamosRemus C, Spindler A, Stanislav M, et al. Baricitinib, methotrexate, or combination in patients with rheumatoid arthritis and no or limited prior disease-modifying antirheumatic drug treatment. Arthritis Rheumatol. 2017;69(3):506-17. https:// doi.org/10.1002/art.39953.

18. Dougados $M$, van der Heijde D, Chen YC, Greenwald M, Drescher E, Liu J, et al. Baricitinib in patients with inadequate response or intolerance to conventional synthetic DMARDs: results from the RA-BUILD study. Ann Rheum Dis. 2017;76(1): 88-95. https://doi.org/10.1136/annrheumdis-2016210094

19. Van De Laar CJ, Oude Voshaar MAH, Fakhouri WKH, Zaremba-Pechmann L, De Leonardis F, De La Torre I, et al. Cost-effectiveness of a JAK1/JAK2 inhibitor vs a biologic disease-modifying antirheumatic drug (bDMARD) in a treat-to-target strategy for rheumatoid arthritis. Clinicoecon Outcomes Res. 2020;12:213-22. https://doi.org/10.2147/ CEOR.S231558.

20. Schlueter M, Finn E, Diaz S, Dilla T, Inciarte-Mundo J, Fakhouri W. Cost-effectiveness analysis of baricitinib versus adalimumab for the treatment of moderate-to-severe rheumatoid arthritis in Spain.
Clinicoecon Outcomes Res. 2019;11:395-403. https://doi.org/10.2147/CEOR.S201621.

21. Weaver AL. The impact of new biologicals in the treatment of rheumatoid arthritis. Rheumatology. 2004;43(Suppl 3):iii17-23. https://doi.org/10.1093/ rheumatology/keh203.

22. Tian L, Xiong X, Guo Q, Chen Y, Wang L, Dong P, et al. Cost-effectiveness of tofacitinib for patients with moderate-to-severe rheumatoid arthritis in China. Pharmacoeconomics. 2020. https://doi.org/ 10.1007/s40273-020-00961-z.

23. 2015 report on Chinese nutrition and chronic disease. 2015. The National Health and Family Planning Commission.

24. Chinese Rheumatology A. 2018 Chinese guideline for the diagnosis and treatment of rheumatoid arthritis. Zhonghua Nei Ke Za Zhi. 2018;57(4): 242-51. https://doi.org/10.3760/cma.j.issn.05781426.2018.04.004.

25. Alemao E, Johal S, Al MJ, Rutten-van MM. Costeffectiveness analysis of abatacept compared with adalimumab on background methotrexate in biologic-naive adult patients with rheumatoid arthritis and poor prognosis. Value Health. 2018;21(2): 193-202. https://doi.org/10.1016/j.jval.2017.05. 020.

26. Lee MY, Park SK, Park SY, Byun JH, Lee SM, Ko SK, et al. Cost-effectiveness of tofacitinib in the treatment of moderate to severe rheumatoid arthritis in South Korea. Clinl Ther. 2015;37(8):1662-76.e2. https://doi.org/10.1016/j.clinthera.2015.07.001.

27. Carlson JJ, Ogale S, Dejonckheere F, Sullivan SD. Economic evaluation of tocilizumab monotherapy compared to adalimumab monotherapy in the treatment of severe active rheumatoid arthritis. Value Health. 2015;18(2):173-9. https://doi.org/10. 1016/j.jval.2014.10.013.

28. Strand V, Miller P, Williams SA, Saunders K, Grant $S$, Kremer J. Discontinuation of biologic therapy in rheumatoid arthritis: analysis from the Corrona RA registry. Rheumatol Ther. 2017;4(2):489-502. https://doi.org/10.1007/s40744-017-0078-y.

29. Strand V, Willianms S, Miller P, Saunders K, Grant S, Kremer J. Op0064 discontinuation of biologic therapy in rheumatoid arithtis (ra): Analysis from the consortium of rheumatology researchers of North America (Coorona) database. Ann Rheum Dis. 2013;72(Suppl 3):A71-2.

30. Brennan A, Bansback N, Reynolds A, Conway P. Modelling the cost-effectiveness of etanercept in adults with rheumatoid arthritis in the UK. 
Rheumatology. 2004;43(1):62-72. https://doi.org/ $10.1093 /$ rheumatology/keg451.

31. Malottki K, Barton P, Tsourapas A, Uthman AO, Liu $\mathrm{Z}$, Routh K, et al. Adalimumab, etanercept, infliximab, rituximab and abatacept for the treatment of rheumatoid arthritis after the failure of a tumour necrosis factor inhibitor: a systematic review and economic evaluation. Health Technol Assess. 2011;15(14):1-278. https://doi.org/10.3310/ hta15140.

32. Wolfe F, Michaud K. The loss of health status in rheumatoid arthritis and the effect of biologic therapy: a longitudinal observational study. Arthritis Res Ther. 2010;12(2):R35. https://doi.org/ 10.1186/ar2944.

33. Wolfe F, Rasker JJ, Boers M, Wells GA, Michaud K. Minimal disease activity, remission, and the longterm outcomes of rheumatoid arthritis. Arthritis Rheum. 2007;57(6):935-42. https://doi.org/10. 1002/art.22895.

34. WHO mortality database. https://www.who.int/ publications/data/data-collection-tools/whomortality-database Accessed 19 July 2020.

35. Diamantopoulos A, Benucci M, Capri S, Berger W, Wintfeld N, Giuliani G, et al. Economic evaluation of tocilizumab combination in the treatment of moderate-to-severe rheumatoid arthritis in Italy. J Med Econ. 2012;15(3):576-85. https://doi.org/10. 3111/13696998.2012.665110.

36. Oppong R, Kaambwa B, Nuttall J, Hood K, Smith $\mathrm{RD}$, Coast J. The impact of using different tariffs to value EQ-5D health state descriptions: an example from a study of acute cough/lower respiratory tract infections in seven countries. Eur J Health Econ . 2013;14(2):197-209. https://doi.org/10.1007/ s10198-011-0360-9.

37. China Medical Bidding website. http://www.eyiyao. org.cn/. Accessed 30 Sep 2020.

38. The People's Bank of China.Exchange rate. [cited 202027 October]. http://www.pbc.gov.cn.

39. National data base. [cited 202027 October]. http:// data.stats.gov.cn/index.htm.

40. Marseille E, Larson B, Kazi DS, Kahn JG, Rosen S. Thresholds for the cost-effectiveness of interventions: alternative approaches. Bull World Health Organ. 2015;93(2):118-24. https://doi.org/10.2471/ BLT.14.138206.

41. Ramiro S, Sepriano A, Chatzidionysiou K, Nam JL, Smolen JS, van der Heijde D, et al. Safety of synthetic and biological DMARDs: a systematic literature review informing the 2016 update of the EULAR recommendations for management of rheumatoid arthritis. Ann Rheum Dis. 2017;76(6): 1101-36. https://doi.org/10.1136/annrheumdis2016-210708.

42. Stevenson M, Archer R, Tosh J, Simpson E, EversonHock E, Stevens J, et al. Adalimumab, etanercept, infliximab, certolizumab pegol, golimumab, tocilizumab and abatacept for the treatment of rheumatoid arthritis not previously treated with disease-modifying antirheumatic drugs and after the failure of conventional disease-modifying antirheumatic drugs only: systematic review and economic evaluation. Health Technol Assess. 2016;20(35):1-610. https://doi.org/10.3310/ hta20350. 